\title{
Téoros
}

Revue de recherche en tourisme

\section{Tourisme et forêt}

\section{Pour une mise en valeur durable}

\section{Marie Lequin}

Volume 25, numéro 3, automne 2006

Tourisme et forêt, visions d'une gestion intégrée

URI : https://id.erudit.org/iderudit/1071012ar

DOI : https://doi.org/10.7202/1071012ar

Aller au sommaire du numéro

Éditeur(s)

Université du Québec à Montréal

ISSN

0712-8657 (imprimé)

1923-2705 (numérique)

Découvrir la revue

Citer ce document

Lequin, M. (2006). Tourisme et forêt : pour une mise en valeur durable. Téoros, 25(3), 3-5. https://doi.org/10.7202/1071012ar

Ce document est protégé par la loi sur le droit d'auteur. L'utilisation des services d'Érudit (y compris la reproduction) est assujettie à sa politique d'utilisation que vous pouvez consulter en ligne.

https://apropos.erudit.org/fr/usagers/politique-dutilisation/
Cet article est diffusé et préservé par Érudit.

Érudit est un consortium interuniversitaire sans but lucratif composé de l’Université de Montréal, l'Université Laval et l'Université du Québec à Montréal. Il a pour mission la promotion et la valorisation de la recherche. https://www.erudit.org/fr/ 


\section{Tourisme et forêt}

\section{Pour une mise en valeur durable}

\section{Marie Lequin}

L'opinion publique est progressivement devenue favorable à une conception dynamique de l'écologie. Respectueuse de l'équilibre naturel des écosystèmes, acceptable pour la collectivité en termes de coûts, la gestion forestière apparaît comme un outil adapté au respect de l'environnement [...]. La forêt est désormais davantage vécue comme un patrimoine ouvert à tous. (L'Homme et la forêt, Engref, Paris, 1989, cité dans Larrère et Nougarède, p. 119)

On se rappellera que la Commission d'Étude sur la gestion de la forêt publique québécoise, constituée le 23 octobre 2003 et présidée par monsieur Guy Coulombe, avait pour mandat de «dresser un état de situation de la gestion des forêts publiques, dans une perspective de gestion intégrée des ressources ", tout en prenant en considération les besoins et les aspirations de la population québécoise et ce, sous différents aspects. Parmi les aspects spécifiques de ce mandat, on trouvait notamment celui-ci :

Examiner les dimensions économiques, environnementales, fauniques, sociales et régionales ainsi que les aspects de régénération, de pérennité, de biodiversité, d'aménagement et de développement durable touchant la protection et la mise en valeur du milieu forestier et de ses ressources. (p. III) ${ }^{1}$

Dans la perspective du tourisme en milieu naturel, nous avons voulu examiner en quoi le rapport de la Commission Coulombe pourrait contribuer au développement et à la mise en valeur du potentiel (éco)touristique de notre patrimoine forestier. Cette voie défendue par la commission, soit celle d'une

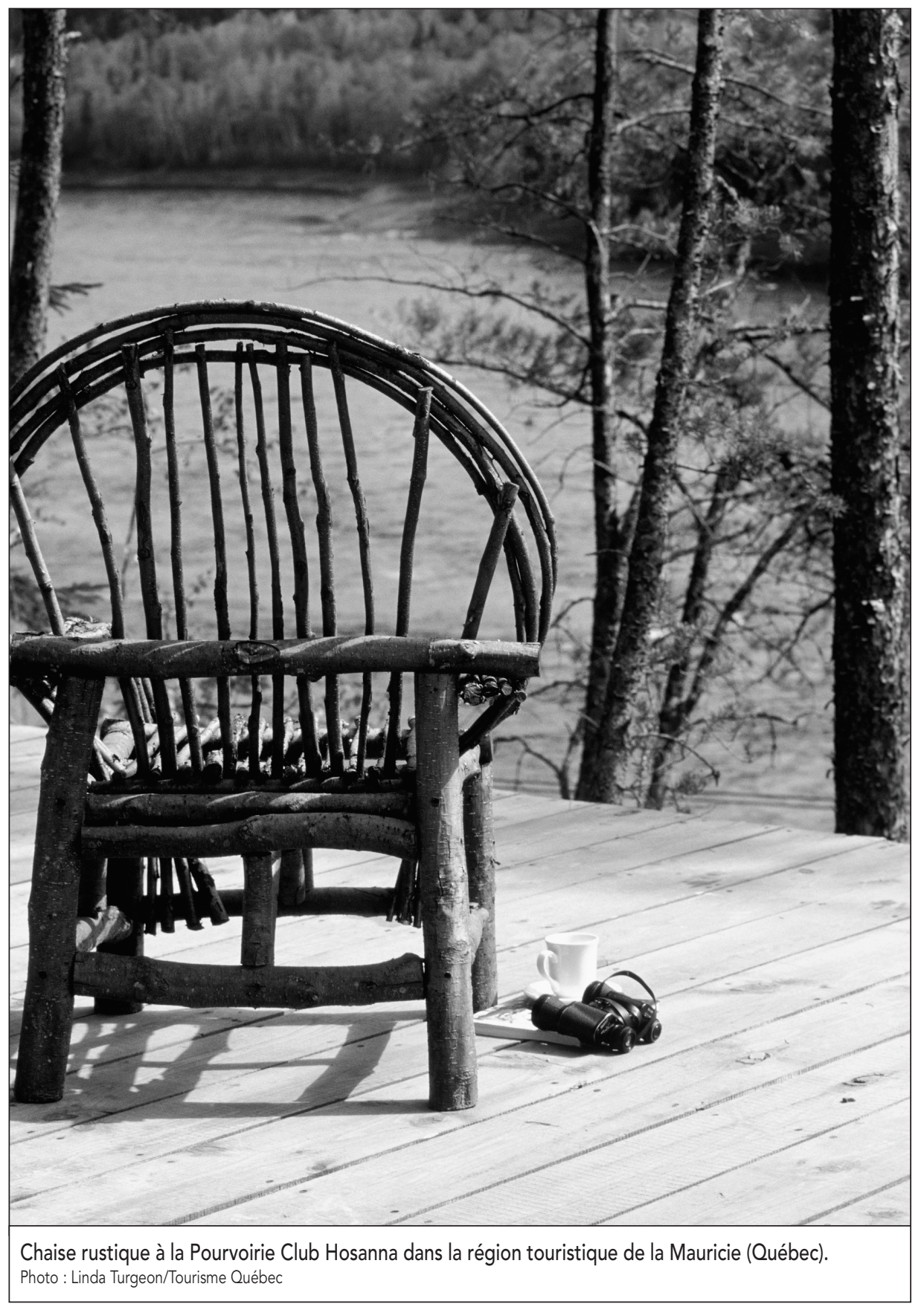


gestion à la fois intégrée et écosystémique des ressources forestières, suscite divers défis et questions, à savoir : Comment cette nouvelle vision de la gestion du plein potentiel forestier entraîne-t-elle la recherche et le développement de nouveaux partenariats avec les intervenants du milieu ? Quel est l'éventail des possibilités de développement et de mise en valeur pour les diverses parties prenantes (publiques et privées) en lien avec le secteur touristique? Comment définir les lieux d'appartenance propres à chacune des parties et leurs limites? Comment instaurer une gestion intégrée de la forêt publique adaptée aux enjeux particuliers à un tel territoire, compte tenu de l'équilibre recherché entre les fonctions économique, écologique et sociale? Enfin, quels nouveaux cadres de gestion écosystémique de l'offre touristique en milieu naturel pourraient être recommandés en vue de faciliter un élargissement de la demande, au-delà des produits traditionnels que sont la chasse et la pêche, ainsi qu'une plus grande accessibilité à la forêt québécoise?

Sans prétendre pouvoir répondre à l'ensemble de ces interrogations, ce numéro de Téoros saisit l'occasion qu'offre la publication de cet important rapport sur l'avenir de la forêt québécoise pour définir, plus précisément, une nouvelle vision du tourisme en milieu forestier fondée sur la gestion intégrée de cette ressource collective. Les diverses contributions sont centrées sur la thématique (éco)tourisme / gestion intégrée / forêt en vue d'approfondir cette dimension implicite mais, en raison du caractère de son mandat, peu développée dans le rapport Coulombe. Notre principal objectif est de faire progresser la réflexion sur la contribution possible du tourisme au développement durable du plein potentiel, autre que ligneux, des écosystèmes forestiers québécois.

Deux thèmes structurants sous-tendent ce dossier: premièrement, celui de la mise en tourisme de la forêt publique et privée selon une approche de gestion intégrée et écosystémique et, deuxièmement, celui de la recherche d'un équilibre d'accessibilité entre les fonctions économique, écologique et sociale, de manière à harmoniser les usages du territoire forestier.

Dans un premier article, G. Beaudet analyse comment les rapports entre l'Homme et la forêt n'ont cessé d'évoluer et de se transformer et ce, depuis le Moyen Âge.

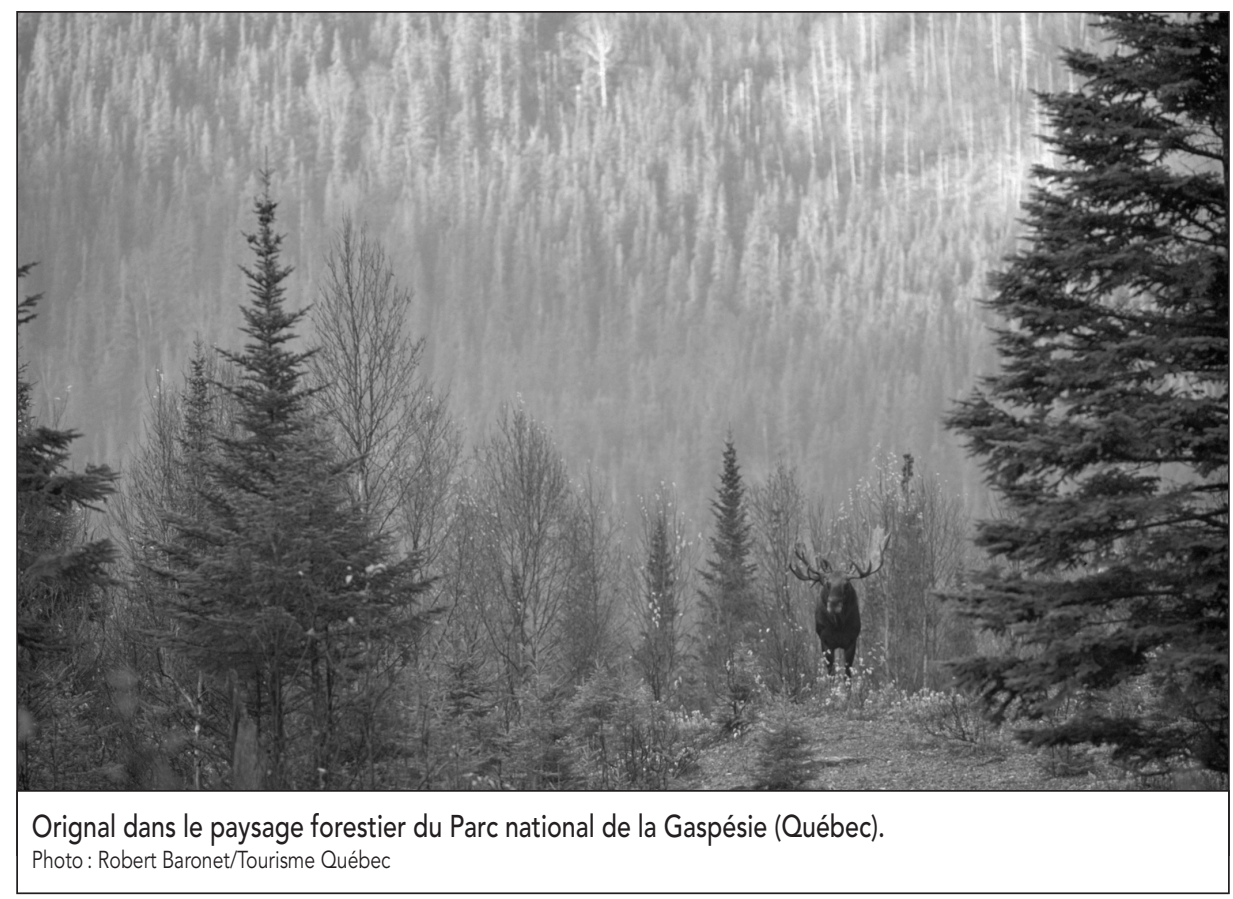

Aujourd'hui hautement recherchée comme lieu de détente et de loisir, la forêt fut tantôt associée à des sentiments d'effroi, de refuge ou encore à un milieu à exploiter. La forêt a toujours occupé une place prépondérante dans l'imaginaire humain comme en témoignent de nombreux contes et légendes. Le texte retrace les origines et l'évolution de notre conception sociale de la forêt et explique comment, " de nos jours, notre désir de nature semble être l'aboutissement d'un parcours qui aurait conduit, à la veille de l'époque romantique, à une réconciliation avec la forêt, ou plutôt à un réenchantement ». Plus récemment, on assiste à une diversité croissante des activités pratiquées en forêt et, par conséquent, à une multiplication des conflits d'usages amenant à reconsidérer la compatibilité des objectifs qui visent d'un côté les usages sociaux et de l'autre les usages économiques de la forêt.

Bien qu'officiellement la politique forestière québécoise mise sur l'aménagement durable de cette ressource, V. Desmarais et L. Bélanger constatent qu'aucune mention n'est faite du tourisme de nature durable sur les terres publiques. En réponse à cette absence, ils ont élaboré un modèle d'encadrement pour un tourisme de nature durable sur les terres publiques du Québec, là où l'exploitation forestière représente un défi substantiel de concordance des usages. Leur article propose une définition conceptuelle du tou- risme durable en contexte forestier québécois, une ressource, pour les auteurs, «trop souvent confondue avec l'écotourisme». Le modèle qu'ils présentent repose sur neuf paramètres de durabilité jugés essentiels pour définir et encadrer le tourisme de nature en milieu forestier. À chacun de ces critères correspondent des éléments qui permettent de préciser les objectifs d'intégration spécifiques devant être atteints. En fait, ce cadre suggère une structure de réflexion valide en vue de doter les territoires forestiers à usages multiples d'une politique de tourisme de nature durable.

L'article de M. Lequin présente un exemple concret de ce que peut être un modèle de gestion intégrée des ressources touristiques en milieu forestier, soit celui de la Société des établissements de plein air du Québec (SÉPAQ) qui a développé, au fil des ans, son propre modèle. Depuis environ une vingtaine d'années, le gouvernement du Québec a graduellement confié à cette société d'État la gestion d'une cinquantaine d'établissements répartis sur l'ensemble du territoire québécois et couvrant une superficie totale de 78600 kilomètres carrés; ils sont regroupés en trois secteurs: les réserves fauniques et SÉPAQ Anticosti (72 166 km²), le réseau des parcs nationaux du Québec $\left(6368 \mathrm{~km}^{2}\right)$ et les centres touristiques $\left(100 \mathrm{~km}^{2}\right)$. L'analyse porte sur la démarche évolutive et émergente de gestion intégrée du tourisme en milieu forestier 
développée par la SÉPAQ en référence à trois axes d'intégration : avec l'appareil de l'État, au sein de l'organisation interne de la SÉPAQ et avec le milieu régional.

L'article de L. Gratton et F. Hone complète le premier thème du dossier en abordant, sous l'angle de la conservation de la biodiversité, les défis de gestion intégrée de la forêt méridionale de tenure privée (représentant 130000 propriétaires). Les auteures montrent que la qualité de la ressource autant que celle du paysage sont intimement liées au développement économique de ces communautés. Bien que la reconnaissance des enjeux économiques, écologiques et sociaux ne soit pas toujours comprise selon la même perspective par l'ensemble des intervenants, elles soulignent qu'un consensus de sensibilité semble se dégager quant aux préjudices subis par la forêt à la suite de différentes pratiques anthropiques «qui ne tiennent pas compte des impacts négatifs sur la biodiversité et la pérennité de la ressource». Elles proposent en outre différents moyens pour préserver ces écosystèmes forestiers privés et contribuer au développement des communautés environnantes, dont: la conservation volontaire, la servitude de conservation forestière, la certification forestière, la diversification des produits et services, ainsi que l'écotourisme.

Le deuxième thème de ce dossier porte sur la question de l'accessibilité qui s'inscrit au cœur des préoccupations et des enjeux posés par la recherche d'un équilibre entre les fonctions économique, écologique et sociale assumées par les territoires forestiers.

L'article de D. Kneeshaw et S. Gauthier rappelle que, avant 1970, l'usage ludique de la forêt était réservé à ceux dont les ressources financières permettaient de se déplacer en hydravion ou en hélicoptère vers leur club privé de chasse et pêche. La problématique du développement historique d'un réseau routier en forêt, y compris la thématique de son accessibilité et des conflits d'usages, est ici analysée sous l'aspect de la modification des processus écologiques, de la planification d'aires protégées et du développement de l'écotourisme. Les auteurs indiquent diverses pistes de conciliation entre les besoins de l'industrie forestière, la demande (éco)touristique et la préservation des écosystèmes, tant pour faciliter l'accès que pour atténuer l'effet des routes en milieu forestier.
L'article de C. Genest montre de façon concrète que le développement d'un vaste réseau de chemins forestiers n'est pas nécessairement garant d'un meilleur accès " public » à la forêt. L'auteur présente différents cas de concertation réussie entre les riverains pour protéger et entretenir leur chemin forestier. II propose de façon vivante une vision sociale élargie de ce que représente un chemin forestier pour un groupe de villégiateurs et soulève l'importance de l'entretien des chemins forestiers voués à des usages diversifiés. À travers le portrait géomorphologique et historique du chemin du Dorval et du lac Fontaine, situés en Mauricie, l'auteur révèle une autre fonction du chemin forestier, celle d'élément de cohésion sociale. II soutient que «le multiusage de la forêt est possible dans des conditions de dialogue avec les usagers, de développement de leur sensibillisation à la richesse de leur environnement naturel, par différentes méthodes », dont une participation démocratique et une gestion intégrée et écosystémique faisant appel à tous les intervenants en cause.

La démocratisation de l'accès aux terres publiques en milieu forestier est également tributaire d'un ensemble de droits accordés par le gouvernement dans le but de générer des activités économiques et sociales d'importance. À ce titre, J. Leblond s'interroge sur la probabilité d'une harmonisation entre les activités (éco)touristiques en milieu naturel public et celles liées à l'exploitation forestière de l'autre. II propose différentes méthodes d'harmonisation des usages, en lien avec les recommandations du Rapport Coulombe et appliquées spécifiquement au cas des pourvoiries à droits exclusifs dont les territoires sont sous contrat d'aménagement et d'approvisionnement forestier (CAAF). Ces outils de planification, telle la simulation visuelle des coupes forestières avant qu'elles ne soient effectuées, sont décrits à partir d'exemples réels et amènent l'auteur à conclure que « la foresterie demeure un puissant outil d'aménagement faunique, récréatif et touristique qui permet de protéger les forêts de catastrophes naturelles comme le feu et les épidémies d'insectes tout en facilitant l'accès à la ressource. »

Dans une perspective de développement touristique d'aires de conservation, certains auteurs se sont intéressés à la mise en œuvre de stratégies de gestion des ac- tivités récréotouristiques assurant le maintien de l'intégrité des processus écologiques. À titre d'exemple, V. Audet, V. Seigner et J. Molard présentent l'étude de suivi de l'intégrité écologique du parc de la forêt ancienne du mont Wright élaborée par l'Association forestière Québec métropolitain, organisme gestionnaire de la mise en valeur et de la sensibilisation de ce parc. Cette étude a permis d'évaluer les impacts sur le milieu naturel liés à la fréquentation du public, principalement lors d'activités de randonnée pédestre et d'escalade. Si les résultats obtenus montrent que l'intégrité écologique du parc est encore relativement bien protégée, les auteurs soulignent que la popularité croissante des activités de plein air exige que l'on se préoccupe dès maintenant de leurs effets potentiels à long terme et suggèrent quelques pistes d'action, dont la sensibilisation du public aux impacts de leur présence.

En guise de conclusion, soulignons que dans la représentation moderne que l'on se fait de la forêt, plus que jamais nature et culture se définissent en complément pour ne pas dire en référent. À preuve, les engagements des Desjardins, Piché, Vigneault, Dupuis, Desrochers et autres, qui prennent ouvertement et activement position pour défendre nos rivières et nos forêts, sont autant de témoignages de notre modernité québécoise qui interpellent notre imaginaire pour des représentations et des usages de la forêt compatibles avec nos aspirations et notre quête de dépassement collectif. Puissent les contributions de ce dossier Téoros être considérées comme une motivation à poursuivre cette vision de l'avenir de la forêt québécoise.

Marie Lequin, rédactrice invitée pour ce dossier, est professeure et directrice des programmes de cycles supérieurs en loisir, culture et tourisme à l'Université du Québec à Trois-Rivières.

\section{Note}

1 [http://www.commission-foret.qc.ca/rapport final.htm].

\section{Bibliographie}

Larrère, R. et O. Nougarède (1993). L'homme et la forêt. Paris : Gallimard. 